\title{
Glucocorticoid-induced alternative promoter usage for a novel 5' variant of granzyme A
}

\author{
Yoshinao Ruike $\cdot$ Susumu Katsuma \\ Akira Hirasawa · Gozoh Tsujimoto
}

Received: 8 November 2006/ Accepted: 22 November 2006/ Published online: 19 December 2006

(C) The Japan Society of Human Genetics and Springer 2006

\begin{abstract}
Glucocorticoids exert diverse physiological functions through transcriptional regulation of genes including granzyme A (GZMA). GZMA is one of the apoptotic effectors localized in cytotoxic $\mathrm{T}$ lymphocytes and is considered to mediate glucocorticoidinduced apoptosis of human leukemia 697 cells. In the present study, we identified a novel $5^{\prime}$ variant transcript of GZMA in dexamethasone (DEX)-treated 697 cells. We designated this novel transcript as GZMA $\beta$. The transcription of GZMA $\beta$ starts at 290 bp downstream of the first intronic glucocorticoid response element (GRE). Chromatin immunoprecipitation assay showed that glucocorticoid receptor (GR) binds to the intronic GRE in a DEX-dependent manner. Luciferase assay and RT-PCR also showed that DEX induces GZMA $\beta$ transcription mediated by GR binding to the intronic GRE. Our results show that there exist at least two transcripts in human GZMA, whose expression is differentially regulated by glucocorticoid.
\end{abstract}

The cDNA sequence of a novel 5' variant of granzyme A (designated as granzyme $\mathrm{A} \beta$; GZMA $\beta$ ) was deposited in GeneBank under number AB284134.

Y. Ruike · S. Katsuma · A. Hirasawa · G. Tsujimoto ( $\square)$

Department of Genomic Drug Discovery Science, Graduate School of Pharmaceutical Sciences,

Kyoto University, 46-29 Yoshida Shimoadachi-cho,

Sakyo-ku, Kyoto 606-8501, Japan

e-mail: gtsuji@pharm.kyoto-u.ac.jp

Present Address:

S. Katsuma

Department of Agricultural and Environmental Biology,

Graduate School of Agricultural and Life Sciences,

University of Tokyo, Tokyo, Japan
Keywords Glucocorticoid · Granzyme A . Leukemia cells · Promoter · GRE

\section{Introduction}

Glucocorticoids are potent immunosuppressive, antiallergic, and anti-inflammatory drugs. Also, synthesized glucocorticoids are widely applicable in medical treatment as chemotherapeutic agents for various leukemias, lymphomas, and multiple myelomas because they efficiently induce apoptosis in lymphoid cells (Amsterdam and Sasson 2002; McManus 2003). Glucocorticoids are known to exert their various effects by regulating transcriptional factor assembly. They can bind and activate the glucocorticoid receptor (GR), and then the activated GR translocates to the nucleus and binds to specific palindromic sequences known as glucocorticoid response element (GRE) (Hayashi et al. 2004). The GR bound to specific GRE has various effects in the gene transcription, and thereby glucocorticoids have a wide range of physiological and therapeutic activities.

Previous DNA microarray studies (Planey et al. 2003; Yoshida et al. 2002; Yamada et al. 2003) have reported the genes responsible for glucocorticoid-induced apoptosis in leukemia 697 cells (Delia et al. 1995), a glucocorticoid-sensitive human pre-B acute lymphocytic leukemia cell line. Among the candidate genes, three studies independently detected the glucocorticoiddependent transcriptional upregulation of granzyme A (GZMA). GZMA belongs to a family of trypsin-like serine proteases localized in cytoplasmic granules of activated lymphocytes and triggers cell death with apoptotic features (Trapani 2001; Lieberman and Fan 
2003; U et al. 2004). Based on the observation that a GZMA inhibitor repressed glucocorticoid-induced apoptosis, we concluded that GZMA is one of the candidate effectors for glucocorticoid-induced apoptosis in 697 cells (Yamada et al. 2003). However, the mechanism by which glucocorticoid induces GZMA remains unclear. More recently, U et al. (2004) investigated the genomic organization of GZMA and identified the consensus GRE in the first intronic region of GZMA gene, speculating the induction potential of intronic GRE to be multiple functions in the context of the complex genomic organization. However, many studies only speculated upon the induction potential of the putative intronic GRE, and lack evidence on the GRE working as an enhancer (U et al. 2004; Kastan et al. 1992; Oda et al. 2000; de Stanchina et al. 2004; Gaunitz et al. 2002). Hence, whether glucocorticoid can induce GZMA via the intronic GRE or not should be more critically assessed. In the present study, we first identified a novel 5' variant transcript of GZMA and the function of a putative intronic GRE in dexamethasone (DEX)-treated 697 cells. By performing chromatin immunoprecipitation (ChIP) assay and reporter gene assay, we found that there exist at least two transcripts in human GZMA, whose expression is differentially regulated by glucocorticoid.

\section{Materials and methods}

\section{Cell culture and treatment}

The 697 cell line is a cloned human pre-B leukemic cell line derived from childhood acute lymphoblastic leukemia that carries the $t(1 ; 19)$ translocation (Findley et al. 1982). Six hundred and ninety seven cells were cultured with RPMI-1640 media supplemented with $10 \%$ fetal bovine serum and $2 \mathrm{mM} \mathrm{L}$-glutamine at $37^{\circ} \mathrm{C}$ under $5 \% \mathrm{CO}_{2}$ in a humidified atmosphere. HepG2 hepatoma cells were cultured with DMEM supplemented with $10 \%$ fetal bovine serum. Dexamethasone was purchased from Sigma Chemical Corporation (St. Louis, MO, USA) and was dissolved in dimethyl sulfoxide (DMSO). DEX was added to the medium at the final concentration adjusted to $100 \mathrm{nM}$. The final concentration of DMSO was adjusted to $0.1 \%(\mathrm{v} / \mathrm{v})$. The control medium contained the same amount of DMSO.

\section{Rapid amplification of $5^{\prime}$ cDNA ends (5'-RACE)}

Six hundred and ninety seven cells $\left(5 \times 10^{6}\right.$ cells $/ 10 \mathrm{ml}$ medium) were incubated for $24 \mathrm{~h}$ in the presence or absence of $100 \mathrm{nM}$ DEX, harvested by centrifugation, and washed in phosphate-buffered saline (PBS). The mRNA was isolated using Micro-FastTrack ${ }^{\circledR}$ (Invitrogen Corp., Carlsbad, CA, USA). Rapid amplification of $5^{\prime}$ cDNA ends (5'-RACE) was carried out using GeneRacer ${ }^{\mathrm{TM}}$ (Invitrogen Corp., Carlsbad, CA, USA). Briefly, an RNA oligonucleotide was selectively ligated to the $5^{\prime}$ ends of decapped mRNA using T4 RNA ligase, and reverse transcribed using oligo dT primers. Gene-specific antisense primers for polymerase chain reaction $(\mathrm{PCR})$ were as follows:

Initial: 5'-AGAATGACCTGGGACCTTTTGTTC-3' Nested: 5'-CCATGTAGGGTCTTGAATGAGGAG TTAC- $3^{\prime}$

Amplified PCR products were subcloned into pGEM-T Easy Vector (Promega Corporation, Madison, WI, USA). Isolated clones were sequenced and searched for corresponding genomic sequence by the BLAST program.

Southern blotting

5'-RACE products were also analyzed by southern blotting. 3'-Labeled oligo-DNA probes for detection of GZMA variants were as follows:

Exon_1a: 5'-GAAACGACAACTGAGAGAGAGG ATGCCAGAAATCTATA-3'

Exon_1b: 5'-CCCTCAGTCCTTTGGTCATTATGA GACTTTTGGTTATT-3'

Probe labeling and signal detection were carried out using Gene Images 3'-oligolabeling and detection module (Amersham Biosciences Corporation, Piscataway, NJ, USA) as suggested by the manufacturer.

\section{Chromatin immunoprecipitation}

ChIP assay was carried out using Chromatin Immunoprecipitation Assay Kit (Upstate Biotechnology, Inc., Lake Placid, NY, USA) as described previously (Katsuma et al. 2005). In brief, 697 cells were treated with or without DEX for $1 \mathrm{~h}$. After crosslinking by adding formaldehyde, cells were washed with PBS, resuspended in SDS lysis buffer, and sonicated to shear genomic DNA. Immunoprecipitation was done by adding an antibody against GR (sc-1002, Santa Cruz Biotechnology, Inc., Santa Cruz, CA, USA) or normal rabbit IgG. After adding protein A agarose, immune complex was washed and co-precipitated DNA fragments were eluted. Recovered DNA fragments were purified by QIAquick PCR purification kit (Qiagen, Valencia, CA, USA) and used as a PCR template. PCR primers for ChIP assays were as follows: 
GZMA_ChIPs: 5'-ACGAAAGGCTTCTATAGCTG-3' GZMA_ChIPas: 5'-AAACTGCACTGTTCTCACTC-3'

Plasmids

DNA fragments containing genomic regions of GZMA (GZMAint1A, GZMAup2k, and GZMAtotal) were amplified by PCR using genomic DNA of 697 cells and were subcloned into pGL3-Basic Vector (Promega). A DNA fragment containing GRE (from +1702 to +2200 , relative to the transcription start site) was prepared from pGL3-GZMAint1A by XbaI digestion. GZMAint1B, GZMAint1C, and GZMAint1D were prepared from pGL3-GZMAint1A by BalI $(+562)$, Bpu1102I (+1141), and EcoRI (+2170) digestion, respectively. GZMA $\triangle$ GRE, a mutant plasmid lacking GRE, was generated by PCR-mediated mutagenesis as described previously (Katsuma et al. 2005). This fragment was inserted into the BamHI site of pGL3GZMAup2k (downstream of luciferase gene) to generate pGL3-GZMAup2k-dE (Fig. 3a). The GREcontaining fragment was also inserted into the NheI site of pGL3 to generate pGL3-uE or the BamHI site of pGL3 to generate pGL3-dE.

\section{Luciferase reporter assay}

Luciferase reporter assay was carried out using DualGlo Luciferase Assay System (Promega Corporation, Madison, WI, USA) as described previously (Katsuma et al. 2005). In brief, approximately $2 \times 10^{5}$ HepG2 cells were grown overnight in $60 \mathrm{~mm}$ dishes and transfected using Lipofectamine Reagent and Plus Reagent (Invitrogen Corp., Carlsbad, CA, USA) with $2 \mu \mathrm{g}$ of reporter constructs, $0.5 \mu \mathrm{g}$ of GR expression vector (pcDNA3 Flag-hGR $\alpha$, a kind gift from Professor Shigeaki Kato, University of Tokyo), and $0.5 \mu \mathrm{g}$ of pRL-TK vector (Promega Corporation, Madison, WI, USA) for an internal control. Twenty-four hours after transfection, cells were treated with DEX $(100 \mathrm{nM})$ for $24 \mathrm{~h}$, and then luciferase activity was measured. The firefly luciferase activity was normalized to the Renilla luciferase activity.

\section{Reverse transcription-PCR (RT-PCR)}

HepG2 cells transfected with reporter plasmids were incubated with or without DEX (100 nM) for $24 \mathrm{~h}$, and total RNA was extracted. First-strand cDNA was synthesized from $1 \mu \mathrm{g}$ of total RNA (DNase-treated) using reverse transcriptase (SuperScript II, Invitrogen Corp., Carlsbad, CA, USA) and was used as a PCR template. PCR primers for semi-quantitative PCR were as follows:

Exon1a_s: 5'-CAGGTTGATTGATGTGGGAC-3'

Exon1b_s: 5'-CATAATGACCAAAGGACTGAG-3' Luciferase_as: 5'-GTGATGTCCACCTCGATATG-3'

\section{Results}

A novel 5' variant type of GZMA is induced by DEX-treatment

To identify the $5^{\prime}$ end of the GZMA transcripts induced by DEX-treatment, we extracted mRNAs from DEX- or DMSO-treated 697 cells and performed RNA ligase-mediated 5'-RACE. Cloned cDNA sequences were then mapped on to the human genomic sequence covering the entire region of GZMA (Fig. 1a). We identified two cDNAs, GZMA $\alpha$ and GZMA $\beta$, which had respective first exons, exon $1 \mathrm{a}$ and exon $1 \mathrm{~b}$, respectively (Fig. 1a, b). GZMA $\alpha$ was identical to the GZMA transcript previously reported (Gershenfeld 1988). GZMA $\beta$ possessed an alternative first exon (exon 1b) and has an open reading frame that encodes an N-terminal variant of GZMA. The transcription start site of GZMA $\beta$ was located in 290 bp downstream region of a putative GRE (Fig. 1a, b).

To verify the expression of GZMA $\beta$, we next examined $5^{\prime}$ terminal fragments of GZMA by Southern blotting of $5^{\prime}$ RACE products using exon $1 \mathrm{a}-$ or exon 1 b-specific oligonucleotide probes. GZMA $\beta$ was induced only when cells were treated with DEX. In contrast to GZMA $\beta$, the expression of GZMA $\alpha$ was detected even without DEX-treatment, and DEXtreatment slightly repressed the expression (Fig. 1c). These results suggest that GZMA have alternative promoters.

GR binds to intronic GRE in vivo and induces GZMA $\beta$

Next, we examined whether the consensus GRE positioned 290 bp upstream of exon $1 \mathrm{~b}$ can function as a promoter for DEX-induced expression of GZMA $\beta$. We performed luciferase reporter assay using HepG2 cells transfected with constructs containing GZMA first intronic fragments. As shown in Fig. 2a, the promoter activity of fragments containing the intronic GRE was significantly upregulated by DEX-treatment. On the other hand, the constructs (GZMAint1D and GZMA $\triangle$ GRE) in which GRE was deleted or mutated were unable to respond to DEX-treatment. Similar 
Fig. 1 Genomic organization of human granzyme $\mathrm{A}$ $(G Z M A)$. a Genomic sequence from the first exon to the second exon. Exons and consensus glucocorticoid response element $(G R E)$ are underlined, and the inferred amino acid sequence is indicated below their corresponding DNA sequences. b Schematic representation of the GZMA genetic locus. White, gray, and black boxes indicate untranslated regions, the leader peptide sequence, and the mature GZMA sequence, respectively. The splicing, represented by solid lines, is only shown between the first and second exons. c Southern blot analysis of amplified 5' cDNA ends. The amplified $5^{\prime}$ cDNA ends were blotted, and the expression of GZMA $\alpha$ or GZMA $\beta$ was detected using exon 1a- or 1b-specific probe, respectively

a

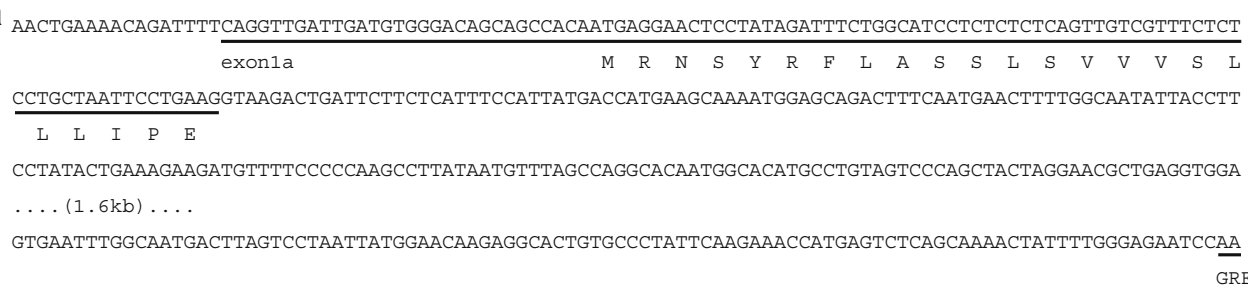
GAACATCTGGTGCAGGAGGTGGAGCACTCGGAGTGTATCACTAAGACATAAGGTTCTCTCTATCTGCATGTATGGTTTGCCTTGTGTTTAGTTCTCACTG TTCCTTAACAATGGGTCTCTACTTCAAGACTATCATCAAGGAAATGTCACTTAGGCAGAATTCTTGGCATATGGTATCTTCTCTCTTCTCTTCCCTATCT CATTGGAAAAGACCTTAGATTAAACCCCATGGTTTGGTCTAGAGATTGAAGGGGAGGTGCAATCGATGTTATTTTCTTAACTTATGAGTGAGAACAGTGC AGTTTAATAACCAAAAGTCTCATAATGACCAAAGGACTGAGGGGTAGGGTCACAGCTAAACGATGCTAATATCGGTTCATAAGCTATATTTTTGCAATGG \begin{tabular}{lllllll}
\hline exon1b & $M$ & T & K & G & L & $R$
\end{tabular}

CTTGGGGTTAAAAGGGAAGTTATGAGGCTTCTAAAAGTCTTATCTATTTCCCCCAAATTAATAGTTAGCCAAAAAGGGTGGGAGCACTTGAGGTTGTCAC TACCACTGGCATTTCCTGCAGCAACTGTTGATGGTAAAGACCAAGGCAGAGCTTTCTGCCCTGGGGCCTGAGGTTGCTGGTTTGTGACATCCAGCCTAAT GATCCTGGAACAGTCAATGGCCTCGCTTCTGTGGGTGGAAAAACAGGACCCTAGAGTCCAACAGGAATAGGATTTGCATCAGATGTCTGCGGGTCTACAT AGAGTAAAAGCAGGGGAAATGCTCTCATCCAGACTTCCTCTCTGAGTGCAAAAAGCATGGCTGGTCTTAGAAACATGACTTTGTGTAGAGCTCTTTGGGG GTGAGCACCAATCTGATTTGTCTTTTTCCATTGAACAGATGTCTGTGAAAAAATTATTGGAGGAAATGAAGTAACTCCTCATTCAAGACCCTACATGGTC $\begin{array}{llllllllllllllllllllllll}\text { exon2 } & D & V & C & E & K & I & I & G & G & D & T & V & V & P & H & S & R & P & Y & M & V\end{array}$ CTACTTAGTCTTGACAGAAAAACCATCTGTGCTGGGGCTTTGATTGCAAAAGACTGGGTGTTGACTGCAGCTCACTGTAACTTGTAAGTGCCTG..... . $\begin{array}{lllllllllllllllllllllllllllllllll}\text { L } & \text { L } & \text { K } & \text { L } & \text { K } & \text { P } & \text { D } & \text { S } & \text { I } & \text { C } & \text { A } & \text { G } & \text { A } & \text { L } & \text { I } & \text { A } & \text { K } & \text { N } & \text { W } & \text { V } & \text { L } & \text { T } & \text { A } & \text { A } & \text { H } & \text { C } & \text { I } & \text { P }\end{array}$

b

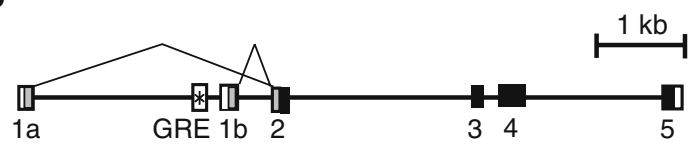

C

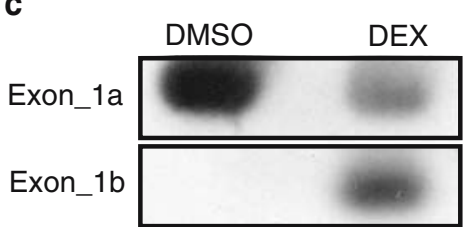

results were also obtained using HeLa cells (data not shown).

Furthermore, we examined whether endogenous GR could physically associate with the intronic GRE by performing ChIP assay. After $1 \mathrm{~h}$ of DEX- or DMSO-treatment, 697 cells were fixed in formaldehyde to crosslink proteins to DNA, and the GR:DNA complexes were precipitated using a GR antibody (da Silva et al. 2003). Following purification, the released DNA was analyzed by PCR using primers that can amplify the putative GRE. The DNA fragments containing putative GRE were abundantly detected in DEX-treated 697 cells, indicating that GR can bind to this region (Fig. 2b). The ChiP assay reproducibly showed an enhanced signal with the GR antibody, two to fivefold stronger than that obtained with control IgG. Taken together, the results show that GZMA $\beta$ expression is directly regulated by GR.

\section{GR differently regulates GZMA $\alpha$ and GZMA $\beta$ expressions}

Next we examined whether GR could transactivate GZMA $\alpha$ by binding to the intronic GRE. To investigate whether GR can activate the upstream promoter region of GZMA $\alpha$, we performed luciferase reporter assay using chimeric constructs that contain the GRE fragment (from +1702 to +2200 , see Fig. 3a) and GZMA $\alpha$ upstream region (from -2331 to +38 ). As shown in Fig. 3a, the GZMA $\alpha$ upstream region has basal promoter activity (pGL3-GZMAup2k), which was eliminated by DEX-treatment regardless of the insertion of GRE fragment into the downstream of luciferase gene (pGL3-GZMAup2k-dE). The plasmid containing only GRE fragment (pGL3-uE) showed DEX-dependent promoter activity, which indicated GRE fragment itself was functional and associates with GR. These results suggest that the GZMA upstream region might be negatively controlled by DEX.

To examine the regulatory function of the intronic GRE, we further performed luciferase reprotor assay by transfecting pGL3-GZMAtotal into HepG2 cells. As shown in Fig. 3b, pGL3-GZMAtotal showed promoter activity with and without DEX-treatment. Also, we extracted mRNAs from HepG2 cells transfected with pGL3-GZMAtotal, and the $5^{\prime}$ sequence of mRNAs were analyzed by RT-PCR. The mRNAs coding luciferase gene were divided into two variants containing either exon $1 \mathrm{a}$ or exon $1 \mathrm{~b}$. The former was substantially repressed by DEX-treatment, whereas the latter was only detectable when the transfected cells were treated with DEX. These results suggest that GR differentially regulates the two GZMA promoters; 


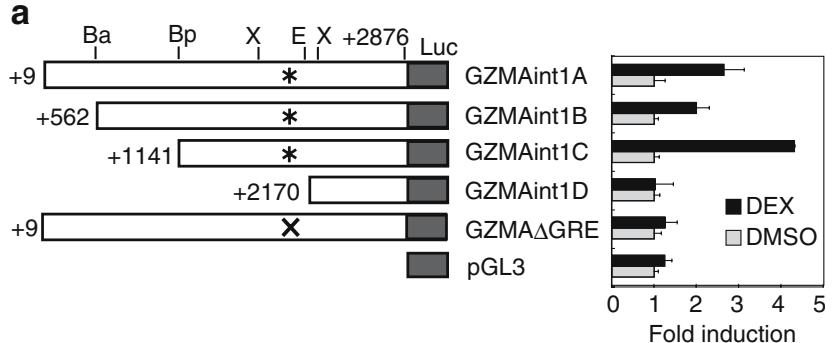

b

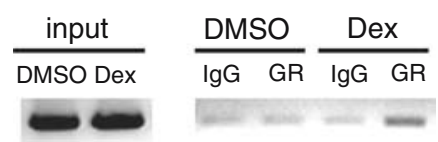

Fig. 2 GZMA first intron contains dexamethasone $(D E X)$ dependent GZMA $\beta$ promoter activity. a In the schematic diagram of GZMA $\beta$ promoter constructs, asterisks indicate the position of consensus GRE (from +1989 to +2005 ). Data show mean \pm SD of three independent experiments. Ba: BalI, Bp: Bpu1102I, X: XbaI, E: EcoRI. b Chromatin Immunoprecipitation $(C h I P)$ assay. Six hundred and ninety seven cells were treated with $100 \mathrm{nM}$ DEX for $1 \mathrm{~h}$. ChIP assay was carried out as described in Materials and methods

thus, GR represses upstream promoter activity while GR directly activates the downstream promoter.

\section{Discussion}

Glucocorticoid receptor bound to specific GRE has a variety of effects on the gene transcription; (a) GR can activate gene transcription by direct binding to GRE adjacent to the promoter, (b) distal enhancers can efficiently activate transcription coupling with proximal enhancer elements, and (c) GR can also indirectly regulate transcription of genes by interacting with other transcription factors (Schoneveld et al. 2004; Rogatsky et al. 1999). Although an increasing number of genes, including the target of GR, were reported to have intronic regulatory elements, the regulatory mechanism is not clear (Kastan et al. 1992; Oda et al. 2000; de Stanchina et al. 2004; Gaunitz et al. 2002). In our study, considering the various potential of the intronic GRE and/or other GREs positioned at distant loci, we investigated transcripts derived from the GZMA gene and for the first time discovered an alternative transcript which starts from 290 bp downstream of GRE. We confirmed the functional properties of the first intronic GRE as a direct regulator of GZMA $\beta$ by finding those observations, (1) reporter constructs containing this element was DEX-responsible (Fig. 2a), and (2) endogenous GR bound to this element (Fig. 2b). On the other hand, GR could not a

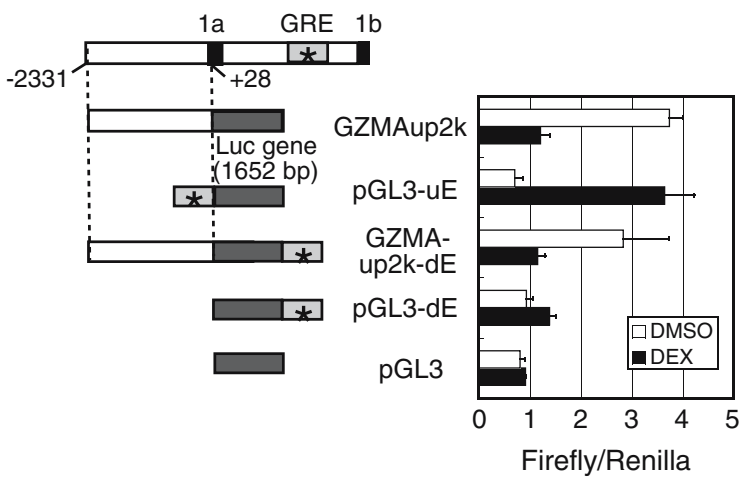

b
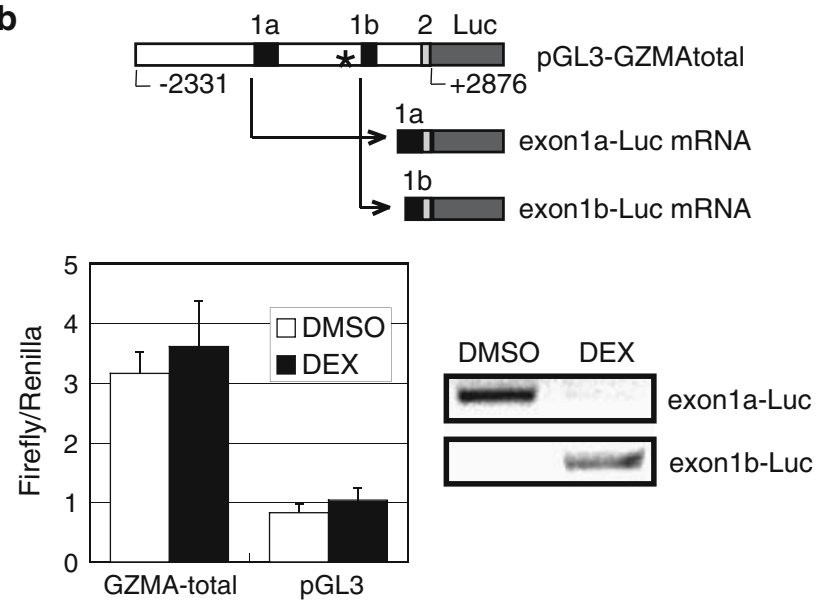

Fig. 3 GZMA $\beta$ but not GZMA $\alpha$ is a direct target of glucocorticoid receptor $(G R)$. a Schematic diagram of GZMA $\alpha$ promoter constructs with or without insertion of GRE-containing fragment (shown as the gray box with an asterisk). Data show mean \pm SD of three independent experiments. b Schematic representation of pGL3-GZMAtotal and the transcripts coding luciferase gene. An asterisk shows the position of GRE. Luciferase reporter assay and semi-quantitative RT-PCR were performed for the same cell line transfected with pGL3-GZMAtotal

enhance but rather repressed upstream promoter activity of the $5^{\prime}$ flanking region of GZMA (Fig. 3a, b). Accordingly, GZMA $\alpha$ was slightly repressed by DEX in 697 cells (Fig. 1c). Taken together, the intronic GRE may act as an operator of promoter usage to switch GZMA first exon in response to DEX-treatment.

Alternative promoters generate two types of GZMA mRNAs with different first exons (exon 1a and 1b). The exon 1a encodes an $\mathrm{N}$-terminal leader peptide, which directs the nascent polypeptide into the lumen of the rough endoplasmic reticulum and is cleaved at the time of packaging into granules (Clark and Griffiths 2003; Jenne and Tschopp 1989). It has been proposed that acidic $\mathrm{pH}$ of the granule environment and/or binding to the granule proteoglycans keeps mature GZMA inactive (Clark and Griffiths 2003; Griffiths and Isaaz 1993). Cytotoxic lymphocytes 
synthesize their own inhibitors that bind and neutralize missorted or mispackaged granzymes to avoid inadvertent cell suicide (Jenne and Tschopp 1989). Active GZMA exerts their apoptotic effects through caspaseindependent mitochondrial damage, cleavage of SET complex, and releasing of GZMA-activated DNase NM23-H1 (Fan et al. 2003a, b; Martinvalet et al. 2005). Because the exon $1 \mathrm{~b}$ encodes a shorter leader peptide with less hydrophobic residues, expression of GZMA $\beta$ may cause atypical subcellular localization of mature GZMA. In fact, the conditioned cell-free medium from DEX-treated 697 leukemia cells contains low levels of GZMA activity, although DEX-treatment induces mRNA expression levels, protein levels, and activity of intracellular GZMA in 697 leukemia cells (Yamada et al. 2003). It is conceivable that DEX-induced GZMA could not be sorted or inactivated correctly in 697 cells. If GZMA $\beta$ mRNA was translated and released as an active GZMA into the cytosol, apoptosisassociated nuclear fragmentation might occur. The glucocorticoid-induced apoptosis of leukemia cells might result from switching of alternative promoter usage that triggers GZMA apoptotic signaling. Interestingly, a splice variant type of mouse GZMA that also result in the alteration of $\mathrm{N}$-terminal leader sequence was reported previously (Hershberger et al. 1992).

Granzyme A has a closest relative gene, granzyme K (GZMK), which is also upregulated by DEX as well as GZMA (Yoshida et al. 2002; Yamada et al. 2003), and the two genes are located only $\sim 70 \mathrm{~kb}$ apart on human chromosome 5. We expected GZMK might be transcriptionally regulated by an alternative promoter as GZMA. Inspection of the DNA sequence over the entire region of GZMK revealed the presence of a candidate GRE positioned at the intronic region (+2930 to +2948). ChIP assay also revealed GR can bind to the GZMK intronic GRE depending upon DEX-treatment, but luciferase reporter assay indicated that it lacks promoter activity with or without DEXtreatment (data not shown). GZMK intronic GRE may have some roles in the glucocorticoid-induced transcriptional activation of GZMK.

In conclusion, by analyzing the $5^{\prime}$-terminal sequence of GZMA transcripts, we discovered a novel 5' variant type of GZMA and demonstrated that GZMA has alternative promoters that are differently regulated by glucocorticoid-treatment in 697 leukemia cells. This study provides a new insight on the GR-mediated transcriptional regulation of GZMA.

Acknowledgments We thank Professor Shigeaki Kato (University of Tokyo) for providing the expression plasmid for GR.
This work was supported in part by research grants from the ScientificFund of the Ministry of Education, Science, and Culture of Japan (to G.T.); the Japan Health Science Foundation and the Ministry of Human Health and Welfare (to G.T.); and the 21st Century Center of Excellence Program "Knowledge Information Infrastructure for Genome Science" (to G.T., S.K. and Y.R.).

\section{References}

Amsterdam A, Sasson R (2002) The anti-inflammatory action of glucocorticoids is mediated by cell type specific regulation of apoptosis. Mol Cell Endocrinol 189:1-9

Clark R, Griffiths GM (2003) Lytic granules, secretory lysosomes and disease. Curr Opin Immunol 15:516-521

Delia D, Aiello A, Formelli F, Fontanella E, Costa A, Miyashita T, Reed JC, Pierotti MA (1995) Regulation of apoptosis induced by the retinoid $N$-(4-hydroxyphenyl) retinamide and effect of deregulated bcl-2. Blood 85:359-367

Fan Z, Beresford PJ, Oh DY, Zhang D, Lieberman J (2003a) Tumor suppressor NM23-H1 is a granzyme A-activated DNase during CTL-mediated apoptosis, and the nucleosome assembly protein SET is its inhibitor. Cell 112:659-672

Fan Z, Beresford PJ, Zhang D, Xu Z, Novina CD, Yoshida A, Pommier Y, Lieberman J (2003b) Cleaving the oxidative repair protein Ape1 enhances cell death mediated by granzyme A. Nat Immunol 4:145-153

Findley Jr HW, Cooper MD, Kim TH, Alvarado C, Ragab AH (1982) Two new acute lymphoblastic leukemia cell lines with early B-cell phenotypes. Blood 60:1305-1309

Gaunitz F, Heise K, Schumann R, Gebhardt R (2002) Glucocorticoid induced expression of glutamine synthetase in hepatoma cells. Biochem Biophys Res Commun 296:10261032

Gershenfeld HK, Hershberger RJ, Shows TB, Weissman IL (1988) Cloning and chromosomal assignment of a human cDNA encoding a $\mathrm{T}$ cell- and natural killer cell-specific trypsin-like serine protease. Proc Natl Acad Sci USA 85:1184-1188

Griffiths GM, Isaaz S (1993) Granzymes A and B are targeted to the lytic granules of lymphocytes by the mannose-6-phosphate receptor. J Cell Biol 120:885-896

Hayashi R, Wada H, Ito K, Adcock IM (2004) Effects of glucocorticoids on gene transcription. Eur $\mathrm{J}$ Pharmacol 500:51-62

Hershberger RJ, Gershenfeld HK, Weissman IL, Su L (1992) Genomic organization of the mouse granzyme A gene. J Biol Chem 267:25488-25493

Jenne DE, Tschopp J (1989) Granzymes: a family of serine proteases in granules of cytolytic T lymphocytes. Curr Top Microbiol Immunol 140:33-47

Kastan MB, Zhan Q, el-Deiry WS, Carrier F, Jacks T, Walsh WV, Plunkett BS, Vogelstein B, Fornace Jr AJ (1992) A mammalian cell cycle checkpoint pathway utilizing p53 and GADD45 is defective in ataxia-telangiectasia. Cell 71:587597

Katsuma S, Ruike Y, Yano T, Kimura M, Hirasawa A, Tsujimoto G (2005) Transcriptional regulation of connective tissue growth factor by sphingosine 1-phosphate in rat cultured mesangial cells. FEBS Lett 579:2576-2582

Lieberman J, Fan Z (2003) Nuclear war: the granzyme A-bomb. Curr Opin Immunol 15:553-559

Martinvalet D, Zhu P, Lieberman J (2005) Granzyme A induces caspase-independent mitochondrial damage, a required first step for apoptosis. Immunity 22:355-370 
McManus R (2003) Mechanisms of steroid action and resistance in inflammation and disease. J Endocrinol 178:1-4

Oda K, Arakawa H, Tanaka T, Matsuda K, Tanikawa C, Mori T, Nishimori H, Tamai K, Tokino T, Nakamura Y, Taya Y (2000) p53AIP1, a potential mediator of p53-dependent apoptosis, and its regulation by Ser-46-phosphorylated p53. Cell 102:849-862

Planey SL, Abrams MT, Robertson NM, Litwack G (2003) Role of apical caspases and glucocorticoid-regulated genes in glucocorticoid-induced apoptosis of pre-B leukemic cells. Cancer Res 63:172-178

Rogatsky I, Hittelman AB, Pearce D, Garabedian MJ (1999) Distinct glucocorticoid receptor transcriptional regulatory surfaces mediate the cytotoxic and cytostatic effects of glucocorticoids. Mol Cell Biol 19:5036-5049

Schoneveld OJ, Gaemers IC, Lamers WH (2004) Mechanisms of glucocorticoid signalling. Biochim Biophys Acta 1680:114-128

da Silva CA, Heilbock C, Kassel O, Frossard N (2003) Transcription of stem cell factor (SCF) is potentiated by glucocorticoids and interleukin-1beta through concerted regulation of a GRE-like and an NF-kappaB response element. FASEB J 17:2334-2336

de Stanchina E, Querido E, Narita M, Davuluri RV, Pandolfi PP, Ferbeyre G, Lowe SW (2004) PML is a direct p53 target that modulates p53 effector functions. Mol Cell 13:523-535

Trapani JA (2001) Granzymes: a family of lymphocyte granule serine proteases. Genome Biol 2:3014.1-3014.7

U M, Shen L, Oshida T, Miyauchi J, Yamada M, Miyashita T (2004) Identification of novel direct transcriptional targets of glucocorticoid receptor. Leukemia 18:1850-1856

Yamada M, Hirasawa A, Shiojima S, Tsujimoto G (2003) Granzyme A mediates glucocorticoid-induced apoptosis in leukemia cells. FASEB J 17:1712-1714

Yoshida NL, Miyashita T, U M, Yamada M, Reed JC, Sugita Y, Oshida T (2002) Analysis of gene expression patterns during glucocorticoid-induced apoptosis using oligonucleotide arrays. Biochem Biophys Res Commun 293:1254-1261 